\title{
HACIA UNA COMPLEJIZACIÓN DE LA TIPOLOGÍA DE FAMILIAS. LOS MODELOS FAMILIARES EN EL NOROESTE ARGENTINO ${ }^{1}$
}

\section{TOWARDS A COMPLEXITY OF FAMILY TIPOLOGIES. THE FAMILY'S MODELS IN NORTHWESTERN ARGENTINA}

\section{Vanesa Vazquez Laba*}

\begin{abstract}
RESUMEN
La investigación teórica-empírica ofrece hallazgos sobre novedosas formas de organización familiar en la región del noroeste argentino. El estudio se centra en la relación trabajo-familia desde una perspectiva que incorpora la división sexual del trabajo familiar como un componente determinante de las estructuras familiares $y$ de sus lógicas de funcionamiento. Se realizó un estudio de caso con familias vinculadas a dos producciones agroindustriales con alto porcentaje de empleo femenino.
\end{abstract}

PALABRAS CLAVES: ARGENTINA * POBLACIÓN RURAL * MERCADO DE TRABAJO * FAMILIA $*$ GÉNERO $*$ TIPOLOGÍA

\section{ABSTRACT}

The research provides theoretical and empirical findings of novel forms of family organization in the region of northwestern Argentina. The study focus on work-family relationship from a perspective that incorporates the gender division of household labor as a determinant of family structures and theirs logic operation. We

1 En el presente trabajo se exponen algunos resultados de la investigación financiada por el CONICET y que concluyó en mi Tesis Doctoral titulada: Desorganizando la tradicional división sexual del trabajo familiar: un estudio comparativo de familias asalariadas rurales del Noroeste Argentino. Algunas ideas y fragmentos también fueron puestos en discusión en la 6ta. Reunión Regional del Grupo "Familias latinoamericanas, pasado y presente: continuidades, transiciones y rupturas", CLACSO, Universidad Nacional de Colombia, Bogotá, 3 al 5 de septiembre de $2008 y$ en el Congress of the Latin American Studies Association, Río de Janeiro, Brazil, June 11-14, 2009.

* $\quad$ Docente de la Facultad de Ciencias Sociales de la Universidad de Buenos Aires. vanesavazquez.laba@gmail.com 
conducted a case study with families linked to agro-industrial production and with high percentage of female employment.

KEYS WORDS: ARGENTINA $*$ RURAL POPULATION $*$ LABOUR MARKET $*$ FAMILY $*$ GENDER $*$ TIPOLOGY

\section{INTRODUCCIÓN}

A partir de la crisis económica y social que se instala en la Argentina desde comienzo de los años 80 , producto de un proceso de transformaciones vinculadas con la reestructuración productiva, la apertura de las importaciones, la privatización de las empresas de servicios públicos y la flexibilización laboral, fundamentalmente, las organizaciones familiares y los modos de vida de sus miembros comienzan a cambiar de forma significativa.

La relación trabajo-familia se transforma de manera radical poniendo en cuestión los modelos de funcionamiento establecidos durante mucho tiempo como "naturales". Tanto la desocupación masculina - principalmente jefes de hogar y jóvenes-, junto a una masiva incorporación de la mano de obra femenina al mercado laboral para aportar ingresos a los deteriorados presupuestos familiares, han sido algunas de las consecuencias del proceso de crisis y cambios sociales.

Asimismo, esta situación ha impactado fuertemente en la definición de las identidades de género $y$ en las prácticas familiares que venían dominando hasta el momento.

Mientras el mundo público y privado estuvieron organizados en función de una estricta segregación genérica no parecía necesario ni pertinente preguntarse por la igualdad de los cónyuges. Estaba claro, para la mayoría, que mujeres $y$ varones eran "naturalmente" distintos $y$, con base en esas diferencias, la sociedad les acordaba roles específicos. El advenimiento de una "nueva mujer" $y$ de un "nuevo varón" también proclamaba por el advenimiento de una "nueva familia". Por tanto, "las identidades rígidas de género habrían sido erosionadas para dar paso a definiciones más flexibles y a prácticas más adaptadas a las necesidades $y$ deseos individuales (Wainerman, 2005: 30).

En las sociedades rurales se comienzan a producir algunos de estos movimientos, particularmente en los años 90, pero adquiriendo características que difieren de las urbanas. Fenómenos tales como la industrializaciónmodernización de las producciones agrícolas; el incremento en los mercados laborales de mano de obra femenina para los productos de exportación; los altos niveles de desocupación o semi-ocupación masculina; la disminución del flujo de los circuitos migratorios internos; entre otros; atravesaron profundamente la institución familiar rural. Al igual que los cambios generados en las familias - redefinición de roles de género; redistribución de las tareas domésticas $y$ de crianza; re-planificación familiar; entre otros-, también han impactado y re-definido las estructuras del empleo y las condiciones laborales en las agroindustrias.

Entonces, desde este contexto de evidentes cambios empíricos el presente artículo se propone, por un lado, recuperar y discutir con las teorías en Ciencias Sociales que plantean análisis unívocos de la relación trabajo-familia (y viceversa); por otro lado, un análisis desde una perspectiva teórica-empírica que plantea la imbricación y el vínculo recíproco entre las esferas del trabajo y la familia, como así también, la existencia de una lógica en común basada en la división del trabajo entre ambos sexos (Barrere-Maurisson, 1999).

Asimismo, el marco teórico adoptado dialogó con los datos empíricos construidos en los trabajos de campo realizados durante cinco años en las provincias de Tucumán y Jujuy, donde se llevaron a cabo observaciones participantes $y$ notas de campo en la zona $y$ entre- 
vistas en profundidad $y$ relatos orales a trabajadoras, trabajadores, empresarios, productores $y$ familiares vinculados a las producciones agroindustriales citrícola y tabacalera.

La selección de los dos casos de estudio se realizó bajo el criterio de que ambas producciones presentan alta participación de fuerza de trabajo femenina en tareas vinculadas con la recolección, la selección y el control de calidad del producto para exportación (Vazquez y Busca, 2003) y porque adquieren rasgos de segregación ocupacional por género (Vazquez Laba, 2008; 2009).

La información se analizó en dos sentidos, por un lado, desde una perspectiva analítica longitudinal apelando a la memoria de los miembros de las familias para reconstruir las historias familiares como historias de caso (Bertaux, 1996), y con el objetivo de poder dar cuenta de las relaciones intrafamiliares a lo largo del tiempo; por otro lado, desde las entrevistas en profundidad y los relatos orales la construcción de una fotografía actual de la dinámica de las formas organizacionales y estructuras de la vida cotidiana. Desde este método de análisis se amplió y mejoró la comprensión del funcionamiento interno de las familias rurales en relación con la división sexual del trabajo familiar.

Uno de los resultados ha sido la construcción de una tipología de familias teniendo en cuenta tres variables fundamentales: una tradicional de las Ciencias Antropológicas, los lazos de parentesco; por otro lado y tomado de la Demografía, la co-residencia o convivencia de las personas en un hogar; y la incorporación novedosa para definir a las familias ha sido incorporar la dimensión de la división familiar del trabajo, rescatada de la Sociología y de los Estudios Feministas.

\section{TRABAJOS Y FAMILIAS EN EL NOROESTE ARGENTINO}

\section{REVISANDO LOS CONCEPTOS PARA PENSAR A LAS} FAMILIAS

Las cuestiones del trabajo y la familia han constituido temas centrales para la Teoría
Social. Ya en la década de 1970 , la producción académica generada en los campos de la Sociología y, principalmente, de la Antropología Económica, se dedicaron, en parte, a la interpretación de los procesos de reproducción social. La relación entre estructuras económicas y estructuras familiares devino, progresivamente, el centro de una reflexión cuyo eje ha sido el trabajo femenino. En una primera instancia, algunos estudios se dedicaron a analizar la relación de las prácticas familiares y el trabajo femenino sólo en la esfera de la producción mercantil, más tarde, se incorporó la indagación de los modos de tratamiento socio-político, jurídico e ideológico de la relación entre la institución familiar y el trabajo de las mujeres.

A partir de la década del 80, los denominados "Estudios de Mujeres" incluyeron a la discusión teórica del momento la sugestiva tesis del valor del trabajo doméstico femenino en el modo de producción capitalista. Autoras como Heidi Hartmann (1984) y Lourdes Benería (1982), recuperaron el concepto de reproducción de la teoría marxista y elaboraron una crítica a las teorías económicas que postulan como improductivo el trabajo doméstico que hacen las mujeres.

Dichos planteos avanzaron en la argumentación evidenciando, a partir del análisis de la situación de las mujeres, que no existe separación del par producción-reproducción, "no hay, pues, una esfera reproductiva separada, de la misma manera que no hay una esfera productiva autónoma, porque la propia existencia de la producción depende de que, a su vez, tenga lugar el flujo constante de su renovación" (Comas D'Argemir, 1995: 26).

Otros modelos explicativos han iniciado una vía diferente de integración entre los pares conceptuales trabajo-familia y producciónreproducción. El antropólogo francés Claude Meillasoux (1998), ha explicitado la relación entre el modo de producción doméstico (ámbito donde se reproduce la fuerza de trabajo por fuera de las normas capitalistas) y el modo de producción capitalista. Destaca la importancia de la esfera doméstica en tanto marco de las relaciones sociales de reproducción, ya que a través del control de la fuerza de trabajo familiar 
-básicamente de las mujeres y mediante el matrimonio-, se perpetúa el sistema económico capitalista.

Las antropólogas feministas, por su lado, han cuestionado fuertemente la tesis de Meillassoux manifestando la ausencia en sus planteos de una explicación sobre las causas de subordinación de las mujeres y su "natural" adscripción a la función reproductiva (Narotzky, 1995).

Por su lado, otros estudios basados en las sociedades latinoamericanas (Stolcke, 1982; 1986), han avanzado sobre el planteo de cómo los sistemas de trabajo hegemónicos locales afectan la estructura de los grupos domésticos familiares modificando, por ejemplo, las pautas de procreación $y$ de crianza, $y$ advierten que estos procesos no se realizan en armonía sino, por el contrario, los cambios socio-económicos en contextos de valores culturales pre-existentes - ya sea como ideología de la familia o como identidades de género-, entran en contradicción permanentemente.

Otra crítica fuerte de los estudios feministas apuntó a la tesis de la teoría económica neoclásica sobre la existencia de una natural línea divisoria entre el "altruismo familiar" de las mujeres y el "interés personal" exclusivo de los varones. Por el contrario, y poniendo en tela de juicio dicha naturalización sostienen que

... El trabajo de cuidado en la familia es a veces impuesto y no altruista [lo que no quiere decir que sea necesariamente penoso o gratificante] y que esa elección está asociada con las relaciones humanas $y$ de poder entre el hombre $y$ la mujer, que también se expresan en el seno del hogar (Amat y León, s.f.: 5).

En relación con esto último, una investigación reciente sobre familia en México plantea el resquebrajamiento de la figura "hombre proveedor masculino" al interior del hogar por el incremento de las familias monoparentales con jefatura femenina, también por el aporte monetario de las mujeres en los núcleos conyugales $y$, principalmente, por la inserción $y$ prolongada participación de la mujer en la actividad económica. Todos estos factores han flexibilizado el rol de proveedor evidenciando una proporción cada vez menor de arreglos formados por la pareja con hijos (arquetipo de la familia nuclear). Hoy en día, los roles sociales son más flexibles, no sólo por la inestabilidad de las situaciones laborales, sino también, por la "actual cultura del consumo y del modernismo, que se tiende a pasar por vivencias (en el sentido fenomenológico de experiencia vivida) cada vez más intensas, experiencias más y más novedosas o más y más profundas" (Salles y Olivo, 2006: 66).

Por tanto, los trabajos mencionados que atraviesan todas las disciplinas de las Ciencias Sociales exponen la necesidad de sobrepasar el análisis unívoco de la relación trabajo-familia o familia-trabajo, ampliando las observaciones y el análisis hacia el interior de ambos ámbitos $y$ en relaciones mutuas e interrogando sobre las diferentes modalidades que conforman dicha conexión.

NUEVAS DINÁMICAS DE LA DIVISIÓN DEL TRABAJO FAMILIAR Y SUS EFECTOS EN LOS MODELOS DE FAMILIA

Históricamente la región del noroeste argentino ha presentado algunas particularidades en la conformación de los mercados laborales, en las condiciones del empleo de la mano de obra y en las estructuras familiares. La producción de la caña de azúcar, hasta mediados del siglo pasado, fue la actividad que dinamizó las económicas provinciales de Jujuy, Salta y Tucumán consolidando, al mismo tiempo, una cultura del trabajo agrícola que repercutió en los vínculos intrafamiliares.

La "cultura cañera" en las familias rurales se fue desdibujando con el advenimiento de la modernización de la agricultura — de la mano de la producción citrícola en la provincia de Tucumán y del tabaco en la provincia de Jujuy-, que incorporó nuevas pautas de producción y de organización laboral que repercutieron en las calificaciones de las personas trabajadoras y en la oferta laboral de la región.

La historia escrita devela la imposición de un modelo familiar occidental-cristiano, patriarcal, monogámico y heterosexual impuesto por la 
conquista de América y regulado por el Concilio de Trento de $1536^{2}$. Dicho modelo penetró en la estructura social colonial, modificando pautas y conviviendo con estilos locales que producirían diversas formas de organización doméstica. Estas formas familiares se inscribieron dentro de los grupos sociales de la época, que se consolidaron a través de la intersección de dos ejes de pertenencia: la "étnico-racial" y la de "clase". Las diversas estrategias matrimoniales, de organización doméstica y de crianza dieron como resultado múltiples estructuras: familias nucleares con amplias redes de parentesco; una alta proporción de uniones consensuales y exogámicas; $y$ un importante porcentaje de familias conducidas por mujeres (López, 1995).

En la actualidad, también se produce una diversidad de formatos familiares a través de los diferentes "arreglos" que realizan los miembros del reparto del trabajo familiar - tanto productivo como reproductivo: familias monoparentales con jefatura femenina; hogares de padre y madre desocupado(a) y trabajo adolescente; cuidado y crianza en manos de hermanos(as), abuelos(as) o vecinos; entre otros.

El modelo de familia nuclear occidental existe en la región con algunas particularidades en relación con el trabajo femenino: algunas mujeres no trabajan y permanecen en los hogares pero otra gran proporción sí trabaja pero sólo cumpliendo un rol secundario como "ayuda familiar" de un miembro varón ${ }^{3}$. En el caso de Tucumán, en la zona estudiada de

En el Concilio de Trento (1536), la Iglesia Católica reforzó las prohibiciones planteadas en el IV Concilio de Letrán (1215), que se basaban en la limitación de las relaciones matrimoniales hasta el cuarto grado de consaguinidad $y$ afinidad parental. En 1937 se produjo una modificación que afectaba directamente a la población aborigen en la que se redujeron las uniones matrimoniales hasta el segundo grado de parentesco (Gil Montero, 2007).
El trabajo femenino en las fincas se realiza bajo la figura de "ayuda familiar" o trabajo "secundario", es decir, las mujeres en esta situación no son contratadas formalmente ni cobran ellas su propia remuneración sino que se hace a través de la persona contactada legalmente que son sus compañeros varones - esposo, padre o hermano.
Tafí Viejo, las familias nucleares tradicionales están vinculadas, en su mayoría, al trabajo de los ex-talleres del ferrocarril o en la actualidad al trabajo fabril de la industria citrícola. Estas estructuras laborales demandan una división del trabajo familiar tradicional donde los varones son los principales proveedores de ingresos y las mujeres se ocupan de las funciones domésticas y de crianza.

En cambio, en Jujuy, en el pueblo de Perico del Carmen, las familias nucleares adquirieron otras características producto de la historia local relacionada con el entrecruzamiento (y enfrentamiento) cultural que se dio entre la cultura conquistadora hispana y la cultura autóctona andina. Así las organizaciones familiares devinieron en estructuras diversas, algunas extensas y otras suprafamiliares semejantes a los ayllus del pueblo incaico ${ }^{4}$.

La vida cotidiana familiar y laboral en la zona tabacalera jujeña se encuentra atravesada por la cultura del patronazgo (Álvarez, 2004); es a través de la figura del "patrón", dueño de las fincas, que se construyen las relaciones laborales $y$ que se interpone en las relaciones

Existen algunos estudios antropológicos (Isla, 1992), que sostienen la tesis sobre la persistencia de la cultura incaica en la estructura social jujeña. La comunidad inca, entendida como una gran unidad de producción y de reproducción-consumo que concentra diferentes núcleos familiares, se ha sostenido en el tiempo articulándose con el desarrollo capitalista agrario de la provincia. Los ayllus se impusieron como forma organizativa social donde transcurría la vida cotidiana de la mayoría de los habitantes. Estos eran asentamientos domésticos que nucleaban parientes consanguíneos y por elección, discriminando por estatus (estamento) $y$ por sexo. No existía igualdad social ni sexual en estas organizaciones, a pesar de las celebraciones a las diosas madres, entre las que la Pachamama ocupaba un lugar primordial. La vida cotidiana de las familias acontecía bajo la lógica de los ayllus $y$ se centraba en el reparto de las distintas tareas domésticas $y$ productivas compartidas entre varones y mujeres como, por ejemplo, la labor de criar animales podía ser realizada indistintamente por cualquiera de los dos sexos. Tampoco parece haber restricciones en la elección de pareja, aunque en los ayllus de mayor jerarquía había presiones efectivas que determinaban algunas preferencias (Barrancos, 2007). 
familiares ${ }^{5}$. Este tipo de masculinidad hegemónica $y$ de ejercicio del poder se reproduce en las familias a través de la figura del jefe del hogar marcando una clara división sexual del trabajo donde el varón queda como único proveedor de ingresos y la mujer como principal responsable de lo doméstico y reproductivo. Pero, sin embargo, estas mujeres han trabajado desde siempre $y$ siguen trabajando en la actividad tabacalera, principalmente en las etapas de pre $y$ post cosecha tratando de conciliar su labor productiva con la vida doméstica-familiar.

Por tanto, a sus roles de madre y esposa se le suma el de trabajadora no reconocida, trabajadora invisible. Para la temporada alta de tabaco estas mujeres suelen ser solicitadas para llevar a cabo las tareas de cuidado de la planta antes de que las hojas sean cosechadas y luego para la etapa pos cosecha en las tareas de encañado y desencañado ${ }^{6}$. Esta situación de empleo encubierto donde ellas trabajan pero quienes están registrados y cobran son sus maridos vuelve a generarles lazos de dependencia tanto en el ámbito laboral como en el familiar. Esta situación entrampa a las mujeres jujeñas obstruyendo las posibilidades de negociación y reformulación de la división del trabajo familiar y vulnera su posibilidad de autonomía personal.

También otros perfiles familiares predominan en la región. Si bien la impronta cañera $y$ el trabajo en los ingenios consolidó un tipo de familia basada en el modelo nuclear otros fenómenos como el trabajo discontinuo zafrero y las migraciones laborales de los varones afectaron los vínculos familiares desintegrando

Es el patrón-padre quien gobierna la finca y a los que trabajan y viven allí. Conoce los vínculos, los hábitos y necesidades cotidianas de las familias que emplea. La forma de dominación es a través del trabajo y de la vivienda. Cautiva la fuerza de trabajo familiar para los picos de cosecha, emplea sólo al jefe varón de la familia y utiliza al resto para las tareas estacionales que demandan mayor fuerza de trabajo.

Las tareas de encañado $y$ desencañado responden al ejercicio de colocar las hojas de tabaco previamente cosechadas y elegidas, alrededor de una caña y de manera enlazada para luego llevarlas a la estufa para que se sequen al calor. a algunas de estas familias y alejándolas de los modelos tradicionales ${ }^{7}$. Estos movimientos en la actualidad generan un modelo al que se lo ha denominado como familia yuxtapuesta.

Este tipo está formado por pequeños núcleos familiares - también definidos en la investigación como "sub-familias"-, que se vinculan de manera superpuesta, alrededor del núcleo original y dentro de un mismo hogar. $\mathrm{Su}$ funcionamiento es dinámico ya que se basa en relaciones cambiantes en relación con la distribución de las tareas y de los roles domésticos, generando distintos "arreglos" circunstanciales intra e inter sub-familiar. Dichas negociaciones dependen, fundamentalmente, de la posición que tenga cada miembro dentro de la comunidad familiar como así también de las posibilidades de empleo y condiciones de trabajo $y$ salariales.

En el panorama laboral citrícola las mujeres adquieren oportunidades concretas de empleo y, evidentemente, esto repercute de forma positiva en la capacidad de negociación que adquieren para repartir el trabajo doméstico-reproductivo. Los "arreglos familiares" que se producen, a veces, quedan sólo en manos de los miembros femeninos del hogar pero, en muchos casos, también lo empiezan a llevar a cabo los varones. Existe por detrás la idea de unir esfuerzos en un contexto donde las mujeres adquieren empleo por sobre las posibilidades de los varones y la reorganización del trabajo familiar es prácticamente obligatorio para poder llevar adelante la vida cotidiana y laboral.

No obstante, las relaciones al interior de estas unidades familiares son complejas; cada "sub-familia" debe organizar su propio reparto del trabajo doméstico, de cuidado y de crianza $y$ del empleo, $y$ en coordinación con los otros pequeños núcleos. Esta dinámica de cooperación lleva a que estas unidades se encuentren

Este modelo se asemeja al tipo de organización que prevaleció en Europa medieval y que la historiografía definió como "familia troncal" (Cicchelli-Pugeault y Cicchelli, 1999). La misma se caracteriza por ser extensa en relación a las generaciones que conviven en el mismo hogar y por la distribución del trabajo familiar entre los diferentes núcleos. 
en permanente situación de construcción de "arreglos" y, por tanto, provoca que las estructuras organizativas sean más flexibles en cuanto a sus roles y estructura.

En la contingencia, mujeres y varones negocian sobre la distribución de las tareas dentro del hogar intercambiando roles en función de la inserción laboral que cada uno(a) pero, al mismo tiempo, las propias necesidades familiares dentro del núcleo influyen a la hora de arreglar quién sale a trabajar y quién se queda en el hogar. Esta dinámica entre los géneros flexibiliza y posibilita cambios y ajustes familiares en una lógica de yuxtaposición de los núcleos familiares dentro del mismo hogar.

En menor medida, también se han captado otros modelos familiares denominados "familias incompletas" o monoparentales femeninas, ya que en su totalidad están gobernadas por mujeres. Estos núcleos, en general, son composiciones numerosas en cuanto a la cantidad de personas que conviven entre hijos(as), otros familiares $\mathrm{u}$ otros no familiares. Lo novedoso es que en relación con los "arreglos" domésticos-reproductivos se generan simultáneamente dos movimientos: uno hacia "adentro" de las familias, donde la distribución de las tareas domésticas se da entre los distintos miembros que conviven en el hogar - madre, hermanas(os) e hijos(as) u otros no familiares; el otro movimiento es hacia "afuera", con la comunidad, principalmente, con las organizaciones que prestan servicios sociales, ya sean comedores comunitarios, parroquias o escuelas. Las familias monoparentales femeninas recurren a estas estrategias del adentro $y$ del afuera porque deben aliviar la carga de lo doméstico y, principalmente, del cuidado de los(as) niños(as) ya que son mujeres que trabajan a tiempo completo y no disponen de recursos económicos para contratar servicios privados.

Asimismo, en dichas familias las mujeres jefas de hogar son las principales proveedoras económicas aunque también pueden existir otros pequeños aportantes. Son empleadas por la actividad citrícola para las tareas de cosecha $y$ en menor medida en los empaques de selección de la fruta. En los períodos de desocupación o semi-ocupación suelen llevar a cabo "estrategias de supervivencia" en el sentido de que generan ingresos a través de la economía informal.

En la provincia de Jujuy, en cambio, no se han captado familias monoparentales con jefatura femenina vinculadas al tabaco, con lo cual, se refuerza la hipótesis sobre la perpetuación de las relaciones familiares tradicionales en esta sociedad como también lo confirma el modelo de familia bajo patrón.

\section{AMPLIANDO LA TIPOLOGÍA DE FAMILIAS. REFLEXIONES FINALES Y NUEVAS PREGUNTAS}

Los esfuerzos por ampliar el conocimiento sobre los procesos de transformación en la familia es una constante dentro de los estudios de las Ciencias Sociales de los últimos tiempos. Nuevos planteos teóricos, métodos y técnicas de captación de información fortalecen las posibilidades de construir más y mejor información sobre un panorama cada vez más cambiante $y$ diverso.

En un primer momento, proliferaron las investigaciones sobre familia urbana poniendo en cuestionamiento el modelo nuclear patriarcal como única estructura y forma de funcionamiento de las clases trabajadoras. Más recientemente, los análisis en el medio rural abordados desde diferentes perspectivas - historia, geografía humana, estudios culturales $y$ perspectivas feministas-, han terminado de colapsar la idea de un único modelo de familia existente en las sociedades contemporáneas. Como se ha demostrado a lo largo del artículo, la incorporación de la dimension analítica de la división familiar del trabajo ha colaborado de manera sustancial en esta empresa de ampliar las posibilidades de construcción de otros tipos familiares.

El estudio de casos en la región del noroeste en la Argentina ha invitado a la reflexión sobre los diversos modos de vida familiar existentes y su vinculación con el mundo laboral local. Se evidenció, también, que la organización de la vida cotidiana se realiza en relación continua con las posibilidades de empleo, los ciclos ocupacionales, las condiciones laborales y salariales que ofrecen las 
agroindustrias como, así también, estas estructuras laborales se organizan y se sustentan a partir de los perfiles familiares de las y los trabajadores.

Ambas provincias manifestaron particularidades en la conformación de las organizaciones familiares. Por un lado, en Tucumán, como se ha visto, coexisten diferentes tipos vinculados con distintas ramas de actividad e historias laborales locales. La cultura relacionada con la zafra de la caña de azúcar consolidó unos modelos de familia basados en la tradicional división sexual del trabajo mientras que, en la actualidad, la citricultura genera otras composiciones más dinámicas que desarman la forma tradicional de organizar el trabajo familiar. Por otro lado, en la zona tabacalera de la provincia de Jujuy, las familias rurales responden predominantemente al modelo patriarcal de patronazgo donde se mantienen relaciones rígidas y se reproducen los roles de sumisión y explotación de las mujeres, con muy pocos márgenes de negociación y de cambios.

Por tanto, la investigación da pruebas que el planteo teórico bidireccional de la relación trabajo-familia ( $y$ viceversa), observando, fundamentalmente, la lógica de la división familiar del trabajo es indispensable para ampliar el conocimiento sobre las nuevas dinámicas y formas de organización familiar en la actualidad. Asumir analíticamente dicha dimensión requirió, asimismo, ahondar sobre las oportunidades y condiciones laborales de los distintos miembros - en tanto puestos, salarios y continuidad del empleo-, pero, al mismo tiempo, también la posición que ocupan en la estructura familiar - relacionado con el parentesco, el sexo, la edad, la autoridad, entre otras. Ambas variables han sido sumamente necesarias para visualizar y mejorar la comprensión sobre quién y en qué grado pueden los miembros dentro de una organización familiar generar capacidades para la negociación y la conciliación entre el trabajo $y$ la familia.

Por último, es importante destacar, también, que el haber incorporado una perspectiva teórica que analiza las relaciones de poder entre los géneros ha echado luz sobre las obsoletas que se pueden volver algunas conceptualizaciones rígidas y que ya no logran captar las particularidades y los cambios de los fenómenos sociales. En consecuencia, la posibilidad de ir incluyendo nuevas categorías de análisis, como por ejemplo, la sexualidad ayudará a complejizar aún más los estudios sobre familia.

\section{BIBLIOGRAFÍA}

Álvarez, Santiago. Leviatán y sus lobos. Violencia y poder en una comunidad de los Andes colombianos. Argentina: Editorial Antropofagia, 2004.

Amat y León, Patricia. De lo cotidiano a lo público: visibilidad y demandas de género. (Texto en línea). En: <http://168.96.200.17/ar/libros/genero/ amat.rtf $>$ [consultado junio de 2006].

Barrancos, Dora. Mujeres en la Sociedad Argentina. Una historia de cinco siglos. Argentina: Editorial Sudamericana, 2007.

Barrère-Maurisson, Marie-Agnès. La división familiar del trabajo. La vida doble. Argentina: Lumen/Humanitas, 2007.

Bertaux, Daniel. "Historias de casos de familias como método para la investigación de la pobreza". Revista de Sociedad, Cultura y Política 1 (1). Buenos Aires, julio 1996: 3-32.

Benería, Lourdes y Sen, Gita. "Acumulación, reproducción y el papel de la mujer en el desarrollo económico: Una revisión de Buserup". Las trabajadoras del agro en América Latina y el Caribe. ACEP, Bogotá: Editorial Presencia, 1982.

Cicchelli-Pugeault, Catherine y Cicchelli, Vincenzo. Las teorías sociológicas de la familia. Argentina: Editorial Nueva Visión, 1999. 
Comas D’Argemir, Dolors. Trabajo, Género, Cultura. La construcción de desigualdades entre hombres y mujeres. España: Editorial Icaria, 1995.

Gil Montero, Raquel. ¿Métodos, modelos y sistemas familiares o historia de la familia? Familia y Diversidad en América Latina. Estudios de casos. (Libro en línea) En: <http://bibliotecavirtual.clacso. org.ar/ar/libros/grupos/robichaux/04Montero.pdf $>$ [consultado mayo 2007].

Hartmann, Heidi. "Capitalismo, patriarcado y segregación de empleos por sexo". Las mujeres y el trabajo. Rupturas conceptuales. España: Editorial Icaria, 1994.

Isla, Alejandro. "Jujuy en el siglo. Estrategias de investigación. Introducción" y "Dos regiones, un origen". Entre el "silencio" y la "furia". Sociedad y articulación en las tierras altas jujeñas. San Salvador de Jujuy: Proyecto ECIRA, Asal, MLA. 1992.

López, Cristina. "El modelo familiar del antiguo régimen y sus representaciones". $C D$ de las I Jornadas de Antropología Rural, Facultad de Filosofía y Letras. Universidad Nacional de Tucumán. Tucumán, 2005.

Meillassoux, Claude. Mujeres, graneros y capitales. México: Siglo XXI, 1998.

Narotzky, Susana. "Mujer, mujeres, género. Una aproximación crítica al estudio de las mujeres en la Ciencias Sociales". Monografías 14. España: CSLC, 1995.

Salles, Vanina y Olivo, Miguel Ángel. "Roles sociales y acción: los riesgos de inestabilidad laboral y los avatares de la figura del proveedor". Teorías sociales $y$ estudios del trabajo: nuevos enfoques. España: Editorial Anthropos, 2006.

Stolcke, Verena. "Los trabajos de las mujeres". Sociedad, subordinación y feminismo III. Debate sobre la mujer en América Latina y El Caribe: Discusiones acerca de la Unidad Producción-Reproducción. Colombia: Editorial ACEP, 1982.

Stolcke, Verena. Cafeicultura. Homens, mulheres e capital (1850-1980). Brasil: Editorial Brasilierte, 1986.

Vazquez Laba, Vanesa y Busca, Vilma. "Una reflexión sobre las formas de conceptualizar el trabajo femenino en el medio rural". En torno de las metodologías: abordajes cualitativos $y$ cuantitativos. Argentina: Editorial Proa XXI, 2003.

Vazquez Laba, Vanesa. "Arte para la vida. Trabajo femenino y formas de desorganización familiar en la localidad de Tafí Viejo, provincia de Tucumán”. [Tesis de Maestría en Investigación en Ciencias Sociales]. Universidad de Buenos Aires, 2007.

Vazquez Laba, Vanesa. "Desorganizando la tradicional división sexual del trabajo familiar: un estudio comparativo de familias asalariadas rurales del Noroeste Argentino". [Tesis Doctoral en Ciencias Sociales]. Universidad de Buenos Aires, 2008.

Wainerman, Catalina. La vida cotidiana en las nuevas familias: ¿una evolución estancada? Argentina: Editorial Lumiere, 2005. 